\title{
Surface-Induced Dissociation from a Liquid Surface
}

\author{
T. Pradeep, S. A. Miller, and R. G. Cooks \\ Department of Chemistry, Purdue University, West Lafayette, Indiana, USA
}

\begin{abstract}
Mass-selected projectile ions in the tens of electronvolt energy range undergo surfaceinduced dissociation upon collision with a liquid perfluorinated polyether (PFPE) surface. The efficiency of translational-to-vibrational (T-V) energy transfer is similar to that observed for a fluorinated self-assembled monolayer (SAM) surface. The thermometer ion W(CO) ${ }_{6}^{+}$. was used to determine an average $\mathrm{T}-\mathrm{V}$ conversion efficiency of $18 \%$ in the collision energy range of $30-50 \mathrm{eV}$. The surface can be bombarded for several hours without displaying any change in the scattered ion products. Ion-surface reactions occur with some projectiles and are analogous to those seen with the fluorinated SAM surface. For example, WF $(m=1-5)$ and $W(C O)_{n} F_{-}^{+}(n=1-2, m=1-2)$ are generated upon collisions of $W(C O)_{6}^{++}$with the PFPE liquid surface. The ion-surface reactions observed suggest that $F$ atoms and/or $\mathrm{CF}_{3}$ groups are accessible for reaction while the oxygen atoms lie below the outermost surface layer. Chemical sputtering of the liquid surface also occurs and yields common fluorocarbon fragment ions, including $\mathrm{CF}_{3}^{+}, \mathrm{C}_{2} \mathrm{~F}_{5}^{+}$, and $\mathrm{C}_{3} \mathrm{~F}_{7}^{+}$and the oxygenated product $\mathrm{CFO}^{+}$. The liquid surface is remarkably free of hydrocarbon impurities. Collisions of the pyrazine and benzene molecular ions, both probes for hydrocarbon impurities, resulted in very little protonated pyrazine or protonated benzene. (J Am Soc Mass Spectrom 1993, 4, 769-773)
\end{abstract}

$\mathrm{P}$ olyatomic ion-surface collisions are a topic of growing interest in mass spectrometry [1-4]. Several types of processes occur, among which are inelastic collisions. A fraction of the translational energy of the projectile ion is converted into internal energy in an inelastic collision. The excess internal energy acquired by the scattered ion may then cause it to dissociate, a process known as surface-induced dissociation ( $\mathrm{SID}_{;}$see eq 1 , where $\mathrm{S}=$ surface, $\mathrm{A}=$ adsorbate, $\mathrm{m}_{1}^{+}=$projectile ion, $\mathbf{m}_{2}^{+}=$fragment ion, $\mathrm{m}_{3}=$ neutral fragment).

$$
\mathrm{m}_{1}^{+} \stackrel{\mathrm{SA}}{\longrightarrow} \mathrm{m}_{2}^{+}+\mathrm{m}_{3}
$$

This allows a surface to be used in tandem mass spectrometry (MS/MS) experiments instead of a target gas to cause collisional activation of a mass-analyzed ion beam. There are several potential advantages of SID [1] over the conventional gas-phase collisionactivated dissociation experiment: (1) the number of collisions is defined. (2) conversion of translational-tovibrational (T-V) energy is efficient, allowing a large internal energy deposition, and (3) the distribution of internal energies is relatively narrow. Interest in these features has led to the implementation of SID on a number of different types of mass spectrometers [5-10].

Address reprint requests to $R$. $G$. Cooks, Department of Chemistry, Brown Laboratories, Purdue University, West Lafayette, IN 47907.
Most of these experiments use low collision energies $(<100 \mathrm{eV})$ and large scattering angles, but in other cases higher energy ions (often in the kiloelectronvolt range) undergo grazing incidence $[6,11-13]$ with surfaces.

It is known that the nature of the surface affects the type and abundance of the scattered ions $[1,3,11]$. In part, this is due to differences in the efficiency of conversion of energy associated with different target surfaces [15-17]. Different surfaces also result in different ion-surface reactions (eq 2), give different chemical sputtering products (eq 3 ), and produce projectile ion neutralization (eq 4) to different extents. Each of these processes may accompany the inelastic scattering channel which leads to SID. Differences in the degree to which

$$
\begin{gathered}
\mathrm{m}_{1}^{+} \stackrel{\mathrm{SA}}{\longrightarrow} \mathrm{m}_{1} \mathrm{~A}^{+} \\
\mathrm{m}_{1}^{+} \stackrel{\mathrm{SA}}{\longrightarrow} \mathrm{m}_{1}+\mathrm{A}^{+} \\
\mathrm{m}_{1}^{+} \stackrel{\mathrm{SA}^{\prime}}{\longrightarrow} \mathrm{m}_{1}
\end{gathered}
$$

different surfaces cause neutralization of the projectile beam have a large effect on the total abundance of scattered ions, and hence on the efficiency of SID. Self-assembled monolayer (SAM) surfaces were introduced recently for SID as well-characterized, readily available, $r_{r}$ and easily cleaned surfaces $[17,18]$. They are especially valuable for fundamental studies because they are flat, uniform in composition, and have welldefined chemical characteristics. However, ion-surface 
reactions (if not SID) are intrinsically destructive of the surface, and it is important that the total ion dose the surface is exposed to be limited to minimize changes in the surface during the course of an experiment [19].

Distinctions are commonly drawn between static and dynamic secondary ion mass spectrometry (SIMS), based on the total ion dose incident on the target surface [20]. In static SIMS, the dose is kept low enough that, to a good approximation, virgin surface is always sampled. This precludes the use of high ion currents and while satisfactory for surface science experiments, it limits applications of SIMS in the characterization of nonvolatile and thermally labile compounds. This limitation in ion dose was effectively eliminated by Barber et al. [21] who introduced the use of liquid matrices for the study of these types of compounds. It is possible to draw an analogy between static SIMS experiments, carried out in the kiloelectronvolt range and involving momentum transfer sputtering collisions to release analytes from the condensed phase, and static SID experiments, involving a much smaller perpendicular cornponent of ion velocity normal to the surface and leading to the collection of the inelastically scattered ion, or its fragmentation or reaction products. Just as the use of liquid surfaces in SIMS [originally in the form of the closely related fast-atom bombardment (FAB) method] lifted the limitation on total ion flux, so it is possible that the use of a liquid surface in SID might have corresponding advantages. Additional advantages are also expected of an appropriate material, including the renewal of the outermost surface as a consequence of the mobility of the liquid, and the fact that the liquid surface will be molecularly flat, an important advantage over ordinary solid surfaces in studies on the dynamics of SID and related processes.

A number of investigators have previously published studies on the mass spectra of perfluoropolyethers [22-24]. In addition, Nathanson and coworkers [25] recently reported the scattering of rare gases from a commercially available liquid perfluorinated polyether (PFPE) with a vapor pressure of $3 \times$ $10^{-10}$ torr. We report experiments in which a film of this material is used as a surface for SID.

\section{Experimental}

The BEEQ instrument used in these experiments has been described previously [26]. It employs a double focusing mass spectrometer to select an ion beam, and deceleration optics to deliver this beam to the surface with a known collision energy at specified incident and scattering angles. The scattered ions are filtered in terms of kinetic energy by using an electric sector and mass analyzed by using a quadrupole mass filter. The incident angle relative to the surface normal and scattering angle relative to the incident beam were set at approximately $55^{\circ}$ and $90^{\circ}$, respectively. The base pressure of the system was $1 \times 10^{-8}$ torr during the present set of experiments.
The PFPE (trade name Krytox 1625, CAS number 60164-51-4), of chemical composition $\mathrm{F}\left[\mathrm{CF}\left(\mathrm{CF}_{3}\right) \mathrm{CF}_{2} \mathrm{O}\right]_{27(\text { ave })} \mathrm{CF}_{2} \mathrm{CF}_{3}$, was obtained from Du Pont (Wilmington, DE). The liquid is very viscous, with a specific gravity $1.86-1.91$ at $24^{\circ} \mathrm{C}$ [27] and was used as received. 'The sample was allowed to sheet across a stainless steel planchet of $16.5 \mathrm{~mm}$ diameter and gravimetric measurements were used to estimate its thickness as $8 \mu \mathrm{m}$. This corresponds to $3300 \mathrm{mono}$ layers, assuming a $24 \AA$ average dimension for the PFPE molecule. The surface did not show any charging, even at a high ion flux $\left(\sim 1 \mathrm{nA} / \mathrm{mm}^{2}\right)$. Degassing from the liquid was not significant and the pressure in the collision chamber was constant throughout the experiment.

The fluorinated SAM surface was supplied by Professor Chris Chidsey, Stanford University, and was prepared as described elsewhere [28].

\section{Results and Discussion}

This investigation covers several properties of the liquid surface as a target in ion-surface collisions. These are (1) T-V energy transfer. This was expected to be similar to the approximately $19 \%$ conversion [16] observed in the case of the fluorinated thioalkane SAM surface, given the chemical similarity of the surfaces. (2) The ion scattering efficiency, that is the total number of scattered ions of all types measured as a fraction of the number of incoming ions. Note that absolute methods of measuring this efficiency are not available but comparative data taken under identical conditions allow an approximate measure of the effects of the different surfaces. (3) The occurrence of ion-surface reactions and of chemical sputtering. Similarities to the processes observed for the fluorinated SAM surface were sought. (4) Finally, it was of interest to determine if the surface could be subjected to high ion fluxes for long periods without significant changes in the scattered ion spectra as the analogy drawn in the Introduction between dynamic and static SIMS/FAB might lead one to expect. In addition, practical issues concerning the purity of the liquid surface, especially the degree to which it is contaminated by adventitious hydrocarbons, were addressed.

In these studies, a number of different projectiles were used. They included: (1) benzene radical cations, used to qualitatively compare the internal energy deposition from the PFPE liquid surface and the fluorinated SAM surfaces, (2) W(CO) ${ }_{6}^{+}$ions, used to estimate the distribution of internal energies deposited in the inelastically scattered projectile ion, (3) $\mathrm{Xe}^{+\cdot}$ ions, used to study the surface composition by chemical sputtering, (4) tungsten radical cations, used to seek ion-surface reactions, (5) pyrazine radical cations, used to test for the presence of adventitious hydrocarbons, and (6) $\mathrm{Cr}(\mathrm{CO})_{6}^{+}$beams, used to test the effects of bombardment with high fluxes for extended periods. 
SID product spectra for benzene radical cation $(\mathrm{m} / \mathrm{z}$ 78) projectiles were used to qualitatively compare the internal energy deposition upon collision at the PFPE liquid surface versus a fluorinated SAM surface. The $30 \mathrm{eV}$ product spectrum for the PFPE liquid surface gave the following characteristic peaks and approximate relative abundances: $m / z 52(100 \%), m / z 39$ (55\%), $m / z 51$ (50\%), $m / z 50$ (35\%), $m / z 63$ (15\%), $m / z 77(10 \%), m / z \quad 78(10 \%), m / z 27(10 \%), m / z 28$ (10\%), $m / z 29(10 \%)$, while all other fragment ions were of less than $<5 \%$ relative abundance. The fluorinated surface, under the same conditions, gave approximate relative abundance of $m / z 52(100 \%), m / z$ $39(80 \%), m / z 51(70 \%), m / z 50$ (40\%), $m / z 63(20 \%)$, $m / z 77(15 \%), m / z 78(15 \%), m / z 27(10 \%), m / z 28$ $(10 \%)$, and $m / z 29(10 \%)$. The similarity in the spectra suggests a strong similarity in internal energy deposition for the two surfaces studied. This prompted a closer look at the T-V energy conversion efficiency of the PFPE liquid surface.

The thermometer ion $\mathrm{W}(\mathrm{CO})_{6}^{+}$was used to estimate the $T-V$ conversion efficiency upon collisions with the liquid PFPE surface. Figure 1 shows the SID spectrum of $W(\mathrm{CO})_{6}^{+}$recorded at $30 \mathrm{eV}$ collision energy. Formation of the base peak, $\mathrm{W}(\mathrm{CO})_{3}^{+\cdot}$ requires that the impacting projectile acquire at least $6.3 \mathrm{eV}$ of internal energy [29]. The SID spectrum can be analyzed to yield the approximate distribution of internal energies [15] and when this is done, the weighted averagc of the distribution is found to be $5.5 \mathrm{eV}$ with a full width-half maximum of $4.4 \mathrm{eV}$. Using the weighted average, the conversion of $\mathrm{T}-\mathrm{V}$ energy is found to be $18 \%$ at this collision energy, for the given incident and scattering angles. This value agrees well with values derived using the same method for the fluorinated SAM surface, where T-V conversion efficiencies of $19 \%$ are recorded under similar conditions [16]. When the collision energy is raised, there is a corresponding increase in the degree of fragmentation, indicating an increase in internal energy deposition. On the other hand, the

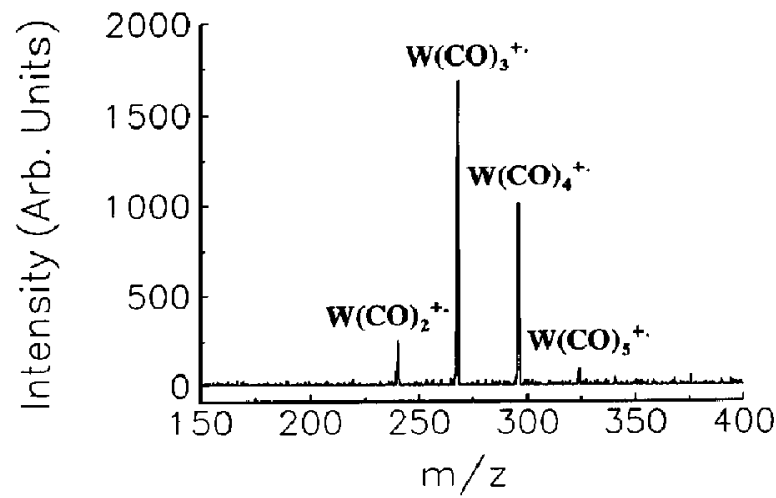

Figure 1. The SID mass spectrum of $\mathrm{W}(\mathrm{CO})_{6}^{++}$at a collision energy of $30 \mathrm{eV}$ for an $8 \mu \mathrm{m}$ thick PFPE surface supported on a stainless steel planchet. Incident and scattering angles were $55^{\circ}$ and $90^{\circ}$, respectively. The ion current was $0.15 \mathrm{nA}$ at the surface. estimated $\mathrm{T}-\mathrm{V}$ efficiency decreases being $18 \%$ at $50 \mathrm{eV}$, $16 \%$ at $70 \mathrm{eV}$, and $12 \%$ at $90 \mathrm{eV}$. The values at the higher energies are less precise because ions due to chemical sputtering begin to make a considerable contribution to the spectrum. For example, the $90 \mathrm{eV}$ spectrum of $\mathrm{W}(\mathrm{CO})_{6}^{+}$on the PFPE surface shows $\mathrm{m} / \mathrm{z}$ $69, \mathrm{CF}_{3}^{+}$, as the base peak and other significant sputtering peaks are $\mathrm{C}_{2} \mathrm{~F}_{5}^{+} \mathrm{m} / z 119(50 \%), \mathrm{C}_{3} \mathrm{~F}_{7}^{+} \mathrm{m} / z 169$ (25\%), while $\mathrm{CF}^{+}, \mathrm{CFO}^{+}, \mathrm{CF}_{2}^{+}, \mathrm{C}_{2} \mathrm{~F}_{3}^{+}, \mathrm{C}_{2} \mathrm{~F}_{4}^{+}, \mathrm{C}_{3} \mathrm{~F}_{5}^{+}$, and $\mathrm{C}_{3} \mathrm{~F}_{6}^{+}$ions are present at low $(<10 \%)$ abundance.

The chemical sputtering process could be studied more easily by scattering $\mathrm{Xe}^{+.}$ions from the surface, and when this was done at $90 \mathrm{eV}$, significant sputtering peaks were $\mathrm{CF}_{3}^{+} m / z 69(100 \%), \mathrm{CF}^{+} m / z 31$ $(50 \%), \mathrm{C}_{2} \mathrm{~F}_{5}^{+} m / z 119(30 \%), \mathrm{C}_{2} \mathrm{~F}_{4}^{+} \mathrm{m} / z 100(25 \%)$, $\mathrm{C}_{3} \mathrm{~F}_{7}^{+} m / z 169(20 \%)$ with other ions of $<5 \%$ relative abundance. The $\mathrm{Xe}^{+}$sputtering spectrum displays close similarities to the sputtering spectrum of the fluorinated SAM surface.

At collision energies above $30 \mathrm{eV}$, the scattered ions resulting from $\mathrm{W}(\mathrm{CO})_{6}^{+}$impact included products of ion-surface reactions (eq 2). These were of the general formulae, WF $(m=1-5)$ and $W(C O))_{11} F^{+}(n=1-2$, $m=1-2)$. The reaction products, $\mathrm{WF}^{+}(m=1-5)$, were also observed when the isotopic $\mathrm{W}^{+}$ion beams $(m / z 184$ and 186) were used as projectiles. The products also contained combinations of $\mathrm{WF}_{ \pm}^{+}$ions with $\mathrm{CF}_{2}$ and $\mathrm{CF}_{3}$ fragments, although in small $(<5 \%)$ relative abundances.

While it is more difficult to quantify the efficiency of a surface with respect to total scattered ion current than to quantify energy transfer efficiency, a relative efficiency was estimated by using the data in Figure 1 and an analogous spectrum from the fluorinated SAM surface. The SID spectrum recorded using the PTPE liquid surface gave approximately five times greater scattered ion current compared to that observed from the fluorinated SAM surface under identical conditions.

Adventitious hydrocarbon impurities can have a significant affect on the appearance of SID spectra, and their possible presence on the liquid surface was investigated by recording the spectrum of pyrazine molecular ion at $30 \mathrm{eV}$ collision energy. Like other heteroaromatic ions, this ion has a great propensity to abstract available hydrogen atoms from molecules present at surfaces [1]. However, the spectrum of the fluorinated liquid showed virtually no hydrogen atom abstraction. Even the relatively clean fluorinated SAM surface shows a much more abundant $(\mathrm{M}+\mathrm{H})^{+}$ion (typical values for the fluorinated SAM surfaces which have been in use for some time are approximately $[M+$ $\mathrm{H}]^{+} / \mathrm{M}^{+}=0.2$ ). The test for adventitious hydrocarbons was repeated after bombardment of both surfaces for 6 hours with $\mathrm{Cr}(\mathrm{CO})_{6}^{+}$. ions with a primary ion current of approximately $1 \mathrm{nA}$. Following the bombardment, the $(\mathrm{M}+\mathrm{H})^{+}$ion was still absent from the PFPE liquid surface but present in the same relative abundance for the fluorinated SAM surface (Figure 2). The 

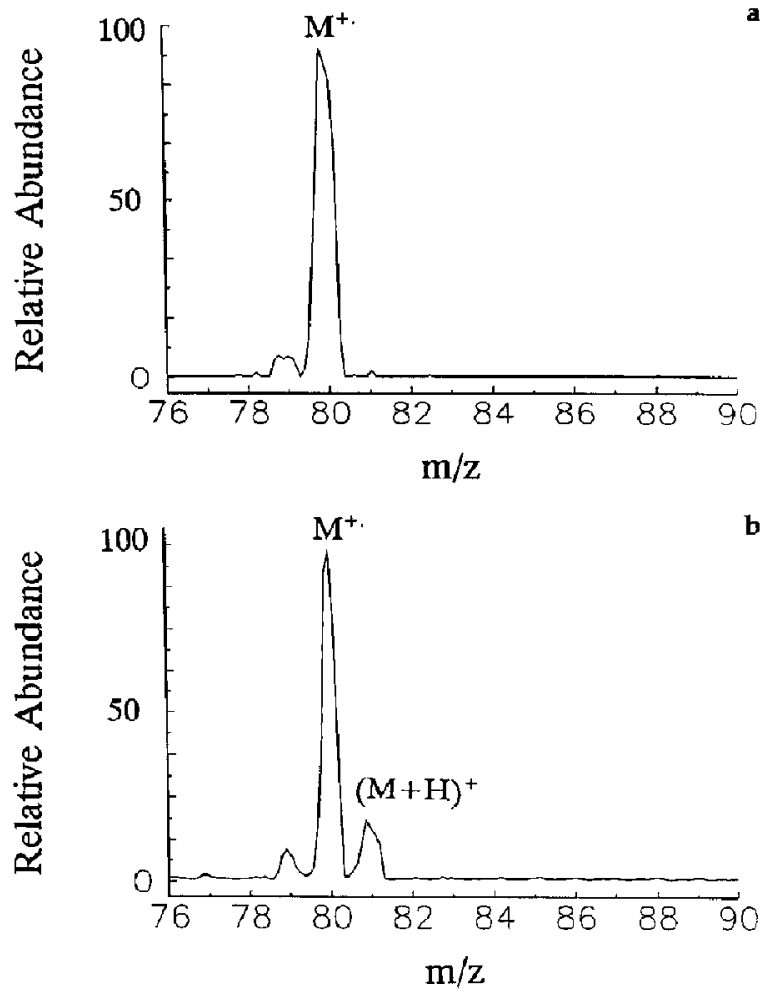

Figure 2. (a) Scattered ions (partial spectrum) recorded when pyrazine molecular ions $(m / z 80)$ impact upon a PFPE liquid surface after 6 hours of bombardment by a $1 \mathrm{nA}$ beam of $\mathrm{Cr}(\mathrm{CO})_{6}^{+}$. Note the $(\mathrm{M}+\mathrm{H})^{+}$ion abundance. (b) The same spectrum for a fluorinated SAM surface recorded under identical conditions.

surface composition following prolonged bombardment of $\mathrm{Cr}(\mathrm{CO})_{6}^{+}$was further investigated by $\mathrm{Xe}^{+\cdot}$ ion sputtering at $90 \mathrm{eV}$, and no change in the product spectrum was observed. Again, hydrocarbon peaks were absent for the PFPF liquid surface; however, for a typical fluorinated SAM surface, hydrocarbon peaks are still seen.

\section{Conclusions}

Several general conclusions follow from this study. First, the need for readily available, higher performance surfaces for SID has at least partly been met. In spite of the attractive features of SID, as listed in the Introduction, the bulk of the work using this technique has been done with surfaces which result in extensive ion beam loss by neutralization as well as lower than optimal T-V conversion energy. Some of these problems were alleviated with the introduction of SAM surfaces. However, the preparation of these surfaces, especially those which are chemically derivatized, is not straightforward, and the fact that they consist of chains a single monolayer in length necessarily makes them somewhat fragile. The use of liquid surfaces, as introduced in this article, makes it possible to prepare stable, well-defined surfaces by simply coating a substrate.

The PFPE liquid surface has potential applications as a SID target in the study of biological molecules. Advantages over other common SID targets include its ease of preparation, large $\mathrm{T}-\mathrm{V}$ conversion efficiency, lack of impurities, and restriction of sputtering peaks to low mass $(<m / z 169)$. Furthermore, the PFPE surfaces can be exposed to high ion fluxes without detectable changes in the spectra. Thin liquid films of PFPE do not charge up and have very similar kinematic properties to the fluorinated SAM surfaces. They also show similar ion-surface reactions. In several respects, further improvements in the properties of liquid surfaces are desirable - the degree of neutralization and the extent of ion-surface reactions are two characteristics in which alternative liquid materials might show even better performance.

In addition to the consequences of the present results for the practice of SID, there are implications for fundamental aspects of ion-surface collisions. First, the fact that these thick liquid films gave similar spectra to those seen for the monolayer fluorinated SAM surfaces suggests that neutralization does not occur by direct tunneling from the substrate. Secondly, the T-V conversion efficiency is controlled by the chemical nature of the outermost layer of surface and not by the structure of the interfacial material. Thus, the greater rigidity of the fluorinated alkyl chains of the fluorinated SAM surface is not the reason why these surfaces show greater $T-V$ conversion than the corresponding hydrogenated SAM surfaces. The reason may lie in the effective mass of the collision partner, made up of a small number of $C$ and $F$ atoms. The energy transfer to the projectile will depend on the ratio of the mass of the ion to the effective mass of the surface; larger energy transfer is possible with the larger effective mass of the fluorinated surfaces, whether a liquid or in the form of a SAM. In addition, the shape of the repulsive potential energy surface will affect the energy transfer efficiency. Third, there is some evidence that these ion-surface collisions involve only direct inelastic scattering. If projectile ion trapping, an alternative process, made a significant contribution, the surface might accumulate a significant number of projectile ions during prolonged bombardment and the trapped species should have been detected through $\mathrm{Xe}^{+\cdot}$ sputtering.

The present experiments appear to be a way to provide new insights into liquid surface structure [30]. The virtual absence of $\mathrm{H}^{-}$atom pick-up in the pyrazine experiment suggests that there are no hydrogen atom (or hydrogen-containing molecule) adsorption sites available on the liquid surface. This, together with the $\mathrm{Xe}^{+}$. sputtering data, suggests that the oxygen atoms are buried below the topmost surface of the liquid. The fact that ion-surface reactions result exclusively in 
fluorine pick-up products also suggests that only $F$ atoms and/or $\mathrm{CF}_{3}$ groups protrude from the outermost layer of the surface [30], consistent with the rare gas scattering results of Nathanson and coworkers [25]. Measurements of the angular dependence of sputtering and SID at the liquid surface are also providing information on the structure of the liquid surface [30].

\section{Acknowledgments}

This work was supported by the National Science Foundation (CI IE 92-23791). We thank Prof. Chris Chidsey and Matt Linford for the self-assembled monolayer surface and acknowledge valuable discussions with Gilbert Nathanson and Ron Macfarlane. We also acknowledge and thank Brian Winger for his contribution in designing and building the BEEQ instrument. T. $P$. is on leave from the CSIR Centre of Excellence in Chemistry, Indian Institute of Science, Bangalore 560 012, India.

\section{References}

1. Cooks, R. G.; Ast, T.; Mabud, Md. A. Int. J. Mass Spectrom. Ion Processes 1990, 100, 209-265.

2. Wright, A. D.; Despeyroux, D.; Jennings, K. R.; Evans, S.; Riddoch, A. Org. Mass. Spectrom. 1992, 27, 525-527.

3. Somogyi, A.; Kane, T. E.; Ding, J.-M.; Wysocki, V. H. J. Am. Chem. Soc. 1993, 115, 5275.

4. Qinyuan, W.; Hanley, L. J. Pkys. Chem. 1993, 97, 2677-2686.

5. Schey, K. L.; Cooks, R. G.; Kraft, A.; Grix, R.; Wollnik, H. Int. J. Mass Spectrom. Ion Processes 1989, 94, 1-14.

6. Aberth, W. Anal. Chem. 1990, 62, 609-611.

7. Li, G.; Duhr, A.; Wollnik, H. I. Am. Soc. Mass. Spectrom. 1992, 3,487 .

8. Williams, E. R.; Jones, G. C.; Fang, L.; Zare, R. N.; Garrison, B. J.: Breiuier, D. W. J. Am. Chem. Soc. 1992, 114, 3207-3210.

9. Ijames, C. F.; Wilkens, C. L. Anal. Chem. 1990, 62, 1295-1299.

10. Lammert, S. A.; Cooks, R. G. I. Am. Soc. Mass Spectrom. 1991, 2, 487-491.

11. Schey, K. L.; Thornburg, K. R. 41st ASMS Conference on Mass Spectrometry and Alied Topics. San Francisco, CA, May 30-June 4 , abstract 75 .
12. Despeyroux, D.; Wright, A. D.; Jennings, K. R.; Evans, S.; Riddoch, A. Int. J. Mass Spectrom. Ion Processes 1992, 122, 133-141.

13. Chorush, R. A.; Vidavsky, I.; McLafferty, F. W. Org. Mass Spectrom, in press.

14. Ast, T,; Srhey, K. I.; Conks, R. G.; I. Serb. Chem. Soc. 1990, 55 , 247-256.

15. DeKrey, M. J.; Kenttämaa, H. I.; Wysocki, V. H.; Cooks, R. G. Org. Mass Spectrom. 1986, 21, 193-195.

16. Morris, M. R.; Riederer, D. E., Jr.; Winger, B. E.; Cooks, R. G.; Ast, T.; Chidsey, C. E. D. Imt. I. Mass Spectrom. Ion Processes 1992, 122, 181-217.

17. Wysocki, V. H.; Jones, J. L.; Ding, J.-M. I. Am. Chem. Soc. $1991,133,8970$.

18. Winger, B. E.; Julian, R. K., Jr.; Cooks, R. G.; Chidsey, C. E. D. J. Am. Chem. Soc. 1991, 113, 8967-8969.

19. Wu, Q.; Hanley, L. 41st ASMS Conference on Mass Spectrometry and Allied Topics. San Francisco, CA, May 30-June 4, abstract 281.

20. Benninghoven, A.; Rüdenauer, F. G.; Werner, H. W. Secondary lon Mass Spectrometry; John Witey \& Sons: New York, 1987.

21. Barber, M.; Bordoli, R. S.; Sedgwick, R. D.; Tyler, A. N. J. Chem. Soc. Chem. Commun. 1981, 325-327.

22. Wilkins, C. L.; Weil, D. A.; Yang, C. L. C.; Ijames, C. F. Anal. Chem. 1985, 57, 520-524.

23. Bletsos, I. V.; Hercules, D. M. Anal. Chem. 1990, 62, 1275-1284.

24. Pacansky, J.; Waltman, R. J. J. Phys. Chem. 1991, 95 , $1512-1518$.

25. King, M. E.; Nathanson, G. M.; Hanning-Lee, M. A.; Minton, T. K. Phys. Rev. Lett. 1993, 70, 1026-1029.

26. Winger, B. E.; Laue, H.-J.; Horning, S. R.; Julian, R. K., Jr.; Lammert, S. A.; Riederer, D. E., Jr.; Cooks, R. G. Rev. Sci. Instrum. 1992, 63, 5613-5625.

27. Material Safety Data Sheet, Dur Pont Chemicals, 5357PP, 1992.

28. Bain, C. D.; Troughton, E. B.; Tao, Y-T; Evall, J.; Whitesides, G. M.; Nuzzo, R. G. J. Am. Chem. Soc. 1989, 111, 321.

29. Winters, R. E.; Kiser, R. W. Inorg. Chem. 1965, 4, 157.

30. Pradeep, T.; Miller, S. A.; Riederer, D. E., Jr.; Cooks, R. G.; in preparation. 